\title{
Evaluation of Geenius HIV-1/2 Confirmatory Assay for the confirmatory and differential diagnosis of HIV-1/HIV-2 in Japan and reliability of the Geenius Reader in the diagnosis of HIV-2
}

Shigeru Kusagawa ${ }^{1 *}$, Ai Kawana-Tachikawa', Keiji Matsubayashi ${ }^{2}$, Yuji Hoshi $^{2}$, Ken Ishimaru ${ }^{3}$ and Isao Hamaguchi ${ }^{4}$

\begin{abstract}
Background: NEW LAV BLOT I and II (LAV I and LAV II), they were only option for human immunodeficiency virus (HIV) confirmatory test, following HIV screening test using HIV Ag/Ab combination test in Japan. We evaluated the performance of Geenius HIV-1/2 Confirmatory Assay (Geenius), both as a confirmatory test and for differentiation between HIV-1 and HIV-2, in comparison with LAV I and LAV II.

Methods: Eighty-nine HIV-1-positive plasma specimens, one anti-HIV-1 low-titer performance panel, 10 seroconversion panels, and two anti-HIV-1/2 combo performance panels were tested. The results were read with the Geenius Reader and by visual reading.

Results: All 89 HIV-1-positive plasma specimens were identified as HIV-1-positive using Geenius; this 100\% success rate was superior to that with LAV I (95.5\% using WHO criteria, 98.9\% using CDC criteria). The HIV-1-positive specimens showed low cross-reactivity with HIV-2 lines in Geenius. The sensitivity of Geenius for HIV-1 detection was the same as or greater than that of LAV I, but less than that of Genscreen HIV Ag-Ab ULT, in our analysis of the commercial performance and seroconversion panels. In contrast, five of the $13 \mathrm{HIV}$-2-positive specimens that had been identified as HIV-positive untypable by visual reading because of their cross-reactivity to HIV-1 lines were successfully identified by the Geenius Reader as HIV-2-positive with cross-reactivity.

Conclusions: Geenius provides strong performance for HIV confirmatory tests and HIV-1 differentiation tests. However, when visual reading is used, its performance in HIV-2 differentiation is less reliable. Because HIV-2 infection has been sporadically reported in Japan, the use of the Geenius Reader is preferable to ensure more reliable HIV-1/HIV-2 differentiation.
\end{abstract}

Keywords: HIV confirmatory test, HIV-1/HIV-2 differentiation test, Sensitivity, Cross-reactivity

\footnotetext{
* Correspondence: kusagawa@nih.go.jp

${ }^{1}$ AIDS Research Center, National Institute of Infectious Diseases, Tokyo, Japan

Full list of author information is available at the end of the article
}

(c) The Author(s). 2021 Open Access This article is licensed under a Creative Commons Attribution 4.0 International License, which permits use, sharing, adaptation, distribution and reproduction in any medium or format, as long as you give appropriate credit to the original author(s) and the source, provide a link to the Creative Commons licence, and indicate if changes were made. The images or other third party material in this article are included in the article's Creative Commons licence, unless indicated otherwise in a credit line to the material. If material is not included in the article's Creative Commons licence and your intended use is not permitted by statutory regulation or exceeds the permitted use, you will need to obtain permission directly from the copyright holder. To view a copy of this licence, visit http://creativecommons.org/licenses/by/4.0/ The Creative Commons Public Domain Dedication waiver (http://creativecommons.org/publicdomain/zero/1.0/) applies to the data made available in this article, unless otherwise stated in a credit line to the data. 


\section{Background}

In Japan, specimens that are detected as human immunodeficiency virus (HIV)-positive via the screening test, using $\mathrm{HIV} \mathrm{Ag/Ab} \mathrm{combination} \mathrm{test,} \mathrm{are} \mathrm{subjected}$ to a confirmatory test using western blot test. The confirmatory test kits, NEW LAV BLOT I and II (LAV I and LAV II), have been used for the last 30 years.

As sporadic cases of HIV-2 infection have been reported in Japan since the 2000s [1-3], the roles of LAV I and LAV II have become more important for HIV-1/ HIV-2 differentiation. However, it is difficult to diagnose HIV-1 or HIV-2 infection based on the results of the LAV I and LAV II tests alone because of cross-reactivity to anti-HIV-2/anti-HIV-1 antibodies, respectively. In our previous study, 12 of 89 (13.5\%) of HIV-1-positive specimens, identified using LAV I and COBAS AmpliPrep/ COBAS TaqMan HIV-1 Version 2.0 (Roche Molecular Systems, Inc., NJ, USA), were identified as HIV-2positive by LAV II testing, and 6 of 13 (46.2\%) of HIV-2positive specimens were identified HIV positive untypable because of the cross-reaction to Env gp160 and Gag p24 of LAV I [4]. According to some reports, HIV-2 infections are often misclassified as HIV-1 while using the HIV-1 western blot [5-7]. In addition, the process of western blotting is complicated, takes a long time, and requires adequate skill in interpreting the results.

The Geenius HIV-1/2 Confirmatory Assay (Geenius) was developed as a confirmatory/supplementary test to improve the diagnostic accuracy of HIV infection. Although the introduction of Geenius in Japan was later than that in American/European countries, it has been available since September 2020. In the US, Geenius has been approved by the FDA and is recommended as a supplementary immunoassay in HIV laboratory testing [8].

In this study, we evaluated Geenius as both a confirmatory test and a differentiation test for HIV-1/HIV-2.

\section{Methods}

\section{HIV-1-positive specimens}

Eighty-nine HIV-1-positive plasma specimens that had been declared ineligible for transfusion were provided by the Japanese Red Cross Blood Centers from 2013 through 2015. The specimens were evaluated using cobas TaqScreen HIV on cobas s 401 (Roche Molecular Systems, Inc., NJ, USA) or Procleix Ultrio Elite ABD assay on Procleix PANTHER System (Grifols Diagnostic Solutions, CA, USA), and decided HIV-1-positive by the discriminatory assay. The specimens were provided following an application for the use of blood donated in Japan, based on the guidelines on the use of donated blood in research and development. The information of the specimens was anonymized, and a decoding index was not created. Ethical approval was obtained from the
Ethical Committee of the National Institute of Infectious Diseases (No. 1082).

\section{Commercial performance panels and seroconversion panels}

Anti-HIV-1 Low Titer Performance Panel PRB107 (SeraCare Life Sciences) and 10 seroconversion panels, namely PRB908, PRB911, PRB913, PRB918 (SeraCare Life Sciences), HIV9012, HIV9015, HIV9032, HIV9077, HIV9079, and HIV12008 (Zeptometrix, Buffalo, NY, USA), were used to evaluate the sensitivity of the assays. Anti-HIV-1/2 Combo Performance Panels PRZ201 and PRZ202 (SeraCare Life Sciences, Milford, MA, USA) were used to evaluate the sensitivity, specificity, and cross-reactivity of the assays between anti-HIV-1 and anti-HIV-2.

\section{Diagnostic HIV tests}

The assays thus evaluated were NEW LAV BLOT I, NEW LAV BLOT II (LAV I and LAV II, Bio-Rad Laboratories, Hercules, CA, USA), and Geenius HIV 1/2 Confirmatory Assay (Geenius, Bio-Rad Laboratories). Each assay was performed according to the manufacturer's instructions. The results of the LAV I assay were primarily interpreted according to the WHO criteria and also according to the CDC criteria [9], as necessary. In brief, two of three Env proteins were required for interpretation of HIV-1-positive in WHO criteria, and two of three proteins, Env gp160/120, Env gp41 and Gag p24, were required for interpretation of HIV-1-positive in CDC criteria. In LAV II test, when one or more Gag, Pol and Env bands were observed, the specimen was interpreted HIV-2-positive. Geenius was read using a Geenius Reader and visual reading according to the instructions in the package insert. In short, when one Env and one or more other HIV-1-specific line(s) were observed, the specimen was decided HIV-1-positive. When two HIV-2-specific lines were observed, the specimen was decided HIV-2-positive. Genscreen ULTRA HIV Ag-Ab (GS ULT, Bio-Rad Laboratories) was used to examine the anti-HIV-1 low titer performance panel and seroconversion panels.

\section{Results}

\section{Examination of HIV-1-positive specimens}

Eighty-nine HIV-1-positive specimens were examined using LAV I and interpreted according to the WHO criteria as recommended in the package insert. This method identified 85 of the specimens (95.5\%) as HIV-1-positive (Table 1). When the CDC criteria were applied, the detection of Env gp160 and Gag p24 in three of the four indeterminate specimens caused them to be identified as HIV1-positive, leading to a total of $88 \mathrm{HIV}$-1-positive specimens (98.9\%) (Table 1). The Geenius assay, by contrast, 
Table 1 Comparison of LAV I with Geenius Assay for HIV-1positive specimens in Japan

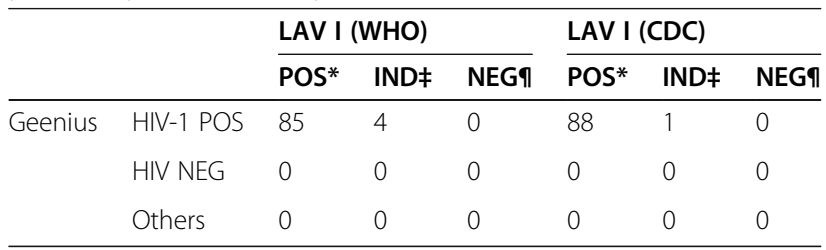

POS*: Positive; IND‡: Indeterminate; NEGI: Negative

identified all 89 specimens as HIV-1-positive (Table 1), whether the results were read using Geenius Reader or by visual reading.

The frequency of each HIV-specific line detected using Geenius is depicted in Table 2. HIV-1 gp160 and gp41 were detected in all tested specimens. The detection frequencies of p31 and p24 were $51.7 \%$. The rates of crossreactivity to two HIV-2 lines were low (1.1 and $4.5 \%$ ), and no specimens reacted with both HIV-2 lines at the same time.

\section{Detection sensitivity of LAV I and Geenius}

The detection sensitivities of LAV I and Geenius were examined. The results of these assays when tested on the anti-HIV-1 Low Titer Performance Panel PRB107 are shown in Table 3. GS ULT identified 14 of the 15 specimens in the panel as HIV-positive (Table 3). These 14 specimens were decided HIV-1-positive by FDAapproved HIV-1 RNA test results according to the datasheet. Six specimens were identified as HIV-negative by LAV I; the same six were also identified as HIV-negative by Geenius (Table 3 (A), (B)). In LAV I, no HIV-1positive results were obtained when the WHO criteria were used (Table $3(\mathrm{~B})$ ); however, when the CDC criteria were used, two specimens were identified as HIV-1positive because of the detection of Env gp160 and Gag p24 (Table $3(\mathrm{C})$ ). In Geenius, five specimens, including the two mentioned above, were identified as HIV-1positive (Table $3(\mathrm{~A})$ ) using both the Geenius Reader and visual reading.

Table 4 shows the measurement using the seroconversion panels. The bleed numbers indicate the blood samples that were first identified as HIV-1-positive. Geenius was able to return HIV-1-positive results earlier than LAV

Table 2 Frequency of HIV-specific line for HIV-1-positive specimens in Japan

\begin{tabular}{|c|c|c|c|c|c|c|}
\hline & \multicolumn{6}{|c|}{ Geenius } \\
\hline & \multicolumn{2}{|l|}{ HIV-2 } & \multicolumn{4}{|c|}{ HIV-1 } \\
\hline & gp36 & gp140 & p31 & gp160 & p24 & gp41 \\
\hline+ & 1 & 4 & 46 & 89 & 46 & 89 \\
\hline- & 88 & 85 & 43 & 0 & 43 & 0 \\
\hline + Rate (\%) & 1.1 & 4.5 & 51.7 & 100 & 51.7 & 100 \\
\hline
\end{tabular}

Table 3 Examination using the anti-HIV-1 Low Titer Performance Panel PRB107

\begin{tabular}{llll}
\hline (A) Geenius HIV 1/2 Confirmatory Assay \\
\hline \multirow{4}{*}{ Geenius } & Genscreen ULTRA HIV Ag-Ab \\
& POS* & NEGף \\
& HIV-1 IND $\neq$ & 3 & 0 \\
& HIV NEG & 6 & 0 \\
& Others & 0 & 1 \\
& & 5 & 0
\end{tabular}

(B) NEW LAV BLOT I (WHO criteria)

\begin{tabular}{llll} 
& & \multicolumn{2}{l}{ Genscreen ULTRA HIV Ag-Ab } \\
& & POS* & NEGף \\
LAV I & POS* & 0 & 0 \\
(WHO) & IND $\neq$ & 8 & 0 \\
& NEGq & 6 & 1
\end{tabular}

(C) NEW LAV BLOT I (CDC criteria)

\begin{tabular}{llll} 
& & \multicolumn{2}{l}{ Genscreen ULTRA HIV Ag-Ab } \\
& & POS* & NEGף \\
LAV I & POS* $^{*}$ & 2 & 0 \\
(CDC) & IND $\neq$ & 6 & 0 \\
& NEGq & 6 & 1 \\
\hline
\end{tabular}

POS*: Positive; IND¥: Indeterminate; NEGI: Negative

I with the WHO criteria but later than LAV I with the CDC criteria (Table 4). The detection sensitivity of these kits was less than that of GS ULT (Tables 3 and 4).

\section{Examination of anti-HIV-1/2 combo performance panels}

The anti-HIV-1/2 Combo Performance Panels were examined using Geenius. The panel includes 13 HIV-1 positive specimens, $13 \mathrm{HIV}-2$ positive specimens, 1 indeterminate specimen, and 3 negative specimens. The frequency of each HIV-specific line detected by Geenius is shown in Table 5. HIV-1-positive specimens showed no

Table 4 Examination using anti-HIV-1 seroconversion panels

\begin{tabular}{|c|c|c|c|c|c|}
\hline \multicolumn{2}{|l|}{ Panel } & \multicolumn{4}{|c|}{ Member of first HIV-1 detection } \\
\hline ID & Bleed & GS ULT & Geenius & LAVI (WHO) & LAVI (CDC) \\
\hline PRB908 & $01-06$ & 06 & 06 & 06 & 06 \\
\hline PRB911(M) & $01-10$ & 02 & 08 & ND & 06 \\
\hline PRB913 & $01-02$ & 02 & 02 & 02 & 02 \\
\hline PRB918 & $01-06$ & 01 & 04 & 04 & 03 \\
\hline HIV9012 & $01-08$ & 06 & $N D^{*}$ & ND & 08 \\
\hline HIV9015 & $01-10$ & 07 & ND & ND & 10 \\
\hline HIV9032 & $01-14$ & 09 & 14 & ND & 10 \\
\hline HIV 9077 & $01-28$ & 12 & 15 & 27 & 15 \\
\hline HIV9079 & $01-25$ & 09 & 14 & 19 & 13 \\
\hline HIV12008 & $01-13$ & 09 & 12 & ND & 11 \\
\hline
\end{tabular}


cross-reactivity to HIV-2-specific lines (Table 5 (A)), whereas approximately $50 \%$ of the HIV-2-positive specimens were cross-reactive to four HIV-1-specific lines (Table 5 (B)). The results obtained by visual reading were the same as those using the Geenius Reader.

The Geenius assay successfully identified 10 of the 13 HIV-1-positive specimens as HIV-1-positive and 11 of the 13 HIV-2-positive specimens as HIV-2-positive, including five that were HIV-2-positive with crossreactivity (Table 6). The five specimens that were HIV-2 positive with cross-reactivity were identified as "HIVpositive untypable" by visual reading because of their cross-reactivity with HIV-1 lines (Table 6). One HIV-2positive specimen was misidentified as HIV-1-positive, since HIV-2 gp140 was not detected (Table 6).

\section{Discussion}

Our study demonstrated the usefulness and characteristics of Geenius. Eighty-nine HIV-1-positive specimens collected in Japan were identified as HIV-1-positive using the Geenius assay, demonstrating a successful detection rate superior to that with LAV I. The detection frequencies of Env gp160, gp120, gp41 and Gag p24 bands were 88/89 (98.9\%), 85/89 (95.5\%), 81/89 (91.0\%), and $89 / 89(100 \%)$, respectively, with LAV I in our previous study [4]. In contrast, the detection rate of two HIV1 Env bands, which are important for HIV-1 diagnosis, was $100 \%$ using Geenius. In addition, 12 of these specimens were identified as HIV-2-positive by LAV II testing because of the cross-reactivity $(12 / 89,13.5 \%)$ [4]. Crossreactivity to Gag p26 (85/89, 96.6\%), Pol p34 (38/89, 42.7\%), Pol p68 (27/89, 30.3\%), and Env gp105 (21/89, 23.6\%) were frequently observed [4]. Geenius showed

Table 5 Frequency of HIV-specific line in anti-HIV-1/2 Combo Performance panels

\begin{tabular}{lllllll}
\hline (A) HIV-1-positive specimens \\
\hline \multicolumn{7}{l}{ Geenius (read by Geenius Reader) } \\
HIV-2 & \multicolumn{7}{l}{ HIV-1 } \\
& gp36 & gp140 & p31 & gp160 & p24 & gp41 \\
+ & 0 & 0 & 4 & 10 & 3 & 11 \\
- & 13 & 13 & 9 & 3 & 10 & 2 \\
+ & 0 & 0 & 30.8 & 76.9 & 23.1 & 84.6
\end{tabular}

(B) HIV-2-positive specimens

\begin{tabular}{lllllll}
\multicolumn{7}{c}{ Geenius (read by Geenius Reader) } \\
& HIV-2 & \multicolumn{5}{c}{ HIV-1 } \\
& gp36 & gp140 & p31 & gp160 & p24 & gp41 \\
+ & 13 & 12 & 6 & 7 & 7 & 7 \\
- & 0 & 1 & 7 & 6 & 6 & 6 \\
+ & 100 & 92.3 & 46.2 & 53.8 & 53.8 & 53.8 \\
\hline
\end{tabular}

Table 6 Examination of anti-HIV-1/2 Combo Performance panels

\begin{tabular}{lllll}
\hline $\begin{array}{l}\text { Geenius } \\
\text { (read by } \\
\text { Geenius } \\
\text { Reader) }\end{array}$ & Datasheet & & \\
\cline { 2 - 5 } & HIV-1 POS* & HIV-2 POS & HIV IND & HIV NEGI \\
\hline HIV POS U† & 0 & 1 & 0 & 0 \\
HIV-1 POS & 10 & 1 & 0 & 0 \\
HIV-2 POS & 0 & 6 & 0 & 0 \\
HIV-2 POS W\$ & 0 & 5 & 0 & 0 \\
HIV IND & 0 & 0 & 0 & 0 \\
HIV-1 IND & 1 & 0 & 0 & 0 \\
HIV-2 IND & 0 & 0 & 0 & 0 \\
NEG & 2 & 0 & 1 & 3 \\
\hline
\end{tabular}

HIV POS U†: HIV-positive untypable; HIV-2 POS W\$: HIV-2 positive with crossreactivity, classified "HIV POS U" by visual reading; POS*: Positive; IND‡: Indeterminate; NEGף: Negative

low cross-reactivity with HIV-2 lines in the examination of the HIV-1-positive specimens.

Geenius exhibited an HIV-1 detection sensitivity comparable to or greater than that of LAV I but lower than that of GS ULT when the results of LAV I were interpreted according to the WHO criteria. Montesinos et al. reported that five of 11 specimens from the acute phase of HIV-1 infection were identified as HIV-1-positive using Geenius [10]. Abbate et al. [11] and Wong et al. [12] reported that the sensitivity of the test for acute HIV-1 infection was low. Kondo et al. reported that seven of 20 specimens from the acute phase of HIV-1 infection were identified as HIV-1-positive [13]. In addition, two of $130 \mathrm{HIV}$-negative specimens and two of 10 Determine HIV-1/2 (Alere Medical, Chiba, Japan)positive and HIV-1 nucleotide amplification testnegative (HIV pseudo-positive) specimens cross-reacted with HIV-1-specific lines [13]. These findings indicate that Geenius should be used for confirmatory/supplementary testing of specimens that tested positive in HIV screening tests.

It has been reported that 46 to $85 \%$ of HIV-2-positive cases in the US were misdiagnosed as HIV-1-positive, based on HIV-1 western blot analysis [5-7]. When HIV2-positive specimens included in the anti-HIV-1/2 Combo Performance Panels, PRZ201 and 202, were tested using LAV I, all 13 specimens were determined HIV-1-indeterminate using LAV I with the WHO criteria. However, the results of six of these specimens (46.2\%) were changed to HIV-1-positive upon interpretation with the CDC criteria because of their crossreactivity to Env gp160 and Gag p24 [4]. Although the detection sensitivity of LAV I with the CDC criteria was higher than that with the WHO criteria, the former was not recommended for discrimination between HIV-1 and HIV-2 infection. 
The cross-reactivity of anti-HIV-2 antibodies to HIV-1 antigens was also observed in the Geenius assay. Six of the 13 HIV-2-positive specimens were identified as HIVpositive untypable by visual reading was used because of cross-reactivity with HIV-1 lines. Five of these six were identified as HIV-2-positive with cross-reactivity when the Geenius Reader was used because the reader could discriminate between specific reactions and crossreactions of anti-HIV-2 antibodies with HIV-1 lines. The positive agreement rate of HIV-2-positive specimens was $6 / 13(46.2 \%)$ by visual reading and $11 / 13(84.6 \%)$ by the Geenius Reader. Herssens et al. reported that six of 10 HIV-2-positive specimens were identified as HIVpositive untypable when visual reading was used in the Geenius assay [14]. In contrast, when the Geenius Reader was used, Malloch et al. reported that 52 out of 53 (98.1\%) HIV-2-positive specimens were identified as HIV-2-positive [15], while Montesinos et al. reported that four out of five HIV-2-positive specimens were identified as HIV-2-positive [10]. These results indicate that Geenius offers strong performance for HIV-2 diagnosis when it is used with the Geenius Reader.

One HIV-2-positive specimen (PRZ202-02) was misidentified as HIV-1-positive using Geenius because of the failure to detect HIV-2 Env gp140, although LAV I/ II with the WHO criteria was able to identify this specimen as HIV-2 positive [4]. Furthermore, clinical reports from HIV-2 epidemic areas should be regularly observed because our data were limited.

Geenius has an strong performance as an HIV confirmatory test; however, its performance when interpreted with visual reading is a cause for some concern. It is important to differentiate between HIV-1 and HIV2 infection because HIV-2 has innate drug resistant mutations for non-nucleoside reverse transcriptase inhibitors and some protease inhibitors [16], and the viral load cannot be quantified to monitor the benefit of antiretroviral therapy using universally distributed HIV-1 RNA quantitation kits. Although HIV-2 infection has been reported only sporadically in Japan, the use of the Geenius Reader is preferable to ensure more reliable HIV-1/2 differentiation. The reader can manage a sample with a bar code on a cassette, save digital capture, and interpret the result automatically. It is also useful to minimize the misidentification of samples, to secure traceability, and to minimize variation in interpretation between individuals.

\section{Conclusions}

Geenius has a strong performance for HIV confirmatory tests and HIV-1 differentiation tests; when visual reading is used, however, its performance in HIV-2 differentiation is less reliable. Almost all cases of HIV infection in Japan are caused by HIV-1. To reduce the cost of equipment introduction, some diagnostic laboratories might opt for the visual reading method. However, HIV2 infection has been sporadically reported in Japan, and correct diagnosis leads to an appropriate choice of treatment. The use of the Geenius Reader is preferable to ensure more reliable HIV-1/HIV-2 differentiation.

\section{Abbreviations}

HIV: Human immunodeficiency virus; LAV: NEW LAV BLOT; WHO: World Health Organization; CDC: Centers for Disease Control and Prevention; FDA: Food and Drug Administration; GS ULT: Genscreen ULTRA HIV Ag-Ab

\section{Acknowledgments}

The HIV-1-positive plasma specimens were provided following an application for the use of blood donated in Japan based on the guidelines on the use of donated blood in research and development. We thank Bio-Rad Laboratories for their cooperation and advice during the conference of the Japan Association of Clinical Reagents Industries.

\section{Authors' contributions}

SK designed the study, performed laboratory analyses, and analyzed the data. SK, AT, KM, YH, and KI prepared the specimens. IH supervised the projects. All authors approved the final manuscript.

\section{Funding}

This work was performed as part of a project for the preparation of reference panels of infectious disease specimens at the National Institute of Infectious Diseases in Japan. This work was also supported by a grant for Research on Regulatory Harmonization and Evaluation of Pharmaceuticals, Medical Devices, Regenerative and Cellular Therapy Products, Gene Therapy Products, and Cosmetics from Japan Agency for Medical Research and Development, AMED.

\section{Availability of data and materials}

The dataset used in this manuscript is available from the corresponding author upon reasonable request.

\section{Declarations}

\section{Ethics approval and consent to participate}

The information of the specimens was anonymized and a decoding index was not created. Ethical approval was obtained from the Ethical Committee of the National Institute of Infectious Diseases (No. 1082).

Consent for publication

Not applicable.

\section{Competing interests}

The authors declare that they have no competing interests.

\section{Author details}

${ }^{1}$ AIDS Research Center, National Institute of Infectious Diseases, Tokyo, Japan. ${ }^{2}$ Central Blood Institute, Blood Service Headquarters, Japanese Red Cross Society, Tokyo, Japan. ${ }^{3}$ Blood Service Headquarters, Japanese Red Cross Society, Tokyo, Japan. ${ }^{4}$ Department of Safety Research on Blood and Biological Products, National Institute of Infectious Diseases, Tokyo, Japan.

Received: 24 November 2020 Accepted: 3 June 2021

Published online: 14 June 2021

\section{References}

1. Kusagawa S, Imamura Y, Yasuoka A, Hoshino H, Oka S, Takebe Y. Identification of HIV type 2 subtype B transmission in East Asia. AIDS Res Hum Retroviruses. 2003;19:1045-9. https://doi.org/10.1089/088922203322 588413 PMID: 14686325.

2. Utsumi T, Nagakawa H, Uenishi R, Kusagawa S, Takebe Y. An HIV-2-infected Japanese man who was a long-term nonprogressor for 36 years. AIDS. 2007; 21:1834-5. https://doi.org/10.1097/QAD.0b013e32827b1477 PMID: 17690592.

3. Ibe S, Yokomaku Y, Shiino T, Tanaka R, Hattori J, Fujisaki S, et al. HIV-2 CRF01_AB:first circulating recombinant form of HIV-2. J Acquir Immune 
Defic Syndr. 2010;54:241-7. https://doi.org/10.1097/QAl.0b013e3181dc98c1 PMID:20502347.

4. Kusagawa S. Evaluation of HIV western blot test kits for HIV confirmatory diagnosis in Japan. Kansenshogaku Zasshi. 2019;93:12-7 (Japanese).

5. Centers for Disease Control and Prevention (CDC). HIV-2 Infection Surveillance-United States, 1987-2009. MMWR Morb Mortal Wkly Rep. 2011; 60:985-8 PMID:21796096.

6. Torian LV, Eavey JJ, Punsalang AP, Pirillo RE, Forgione LA, Kent SA, et al. HIV type 2 in New York City, 2000-2008. Clin Infect Dis. 2010;51:1334-42. https://doi.org/10.1086/657117 PMID:21039219.

7. Nasrullah M, Ethridge SF, Delaney KP, Wesolowski LG, Granade TC, Schwendemann J, et al. Comparison of alternative interpretive criteria for the HIV-1 Western blot and results of the Multispot HIV-1/HIV-2 Rapid Test for classifying HIV-1 and HIV-2 infections. J Clin Virol. 2011;52(Suppl 1):S23-7. https://doi.org/10.1016/j.jcv.2011.09.020 PMID:21993309.

8. Centers for Disease Control and Prevention and Association of Public Health Laboratories. Laboratory testing for the diagnosis of HIV infection: updated recommendations. 2014. Available from: https://doi.org/10.15620/cdc.23447.

9. Centers for Disease Control and Prevention. Interpretive criteria used to report Western blot results for HIV-1-antibody testing--United States. MMWR Morb Mortal Wkly Rep. 1991;40:692-5. PMID:1656186.

10. Montesinos I, Eykmans J, Delforge ML. Evaluation of the Bio-Rad Geenius HIV-1/2 test as a confirmatory assay. J Clin Virol. 2014;60:399-401. https:// doi.org/10.1016/j.jcv.2014.04.025 PMID:24932737.

11. Abbate I, Pergola C, Pisciotta M, Sciamanna R, Sias C, Orchi N, et al. Evaluation in a clinical setting of the performances of a new rapid confirmatory assay for HIV1/2 serodiagnosis. J Clin Virol. 2014;61:166-9. https://doi.org/10.1016/j.jcv.2014.06.015 PMID:25037532.

12. Wong C, Lim S, Tan C, Lui S, Lee Y, Chan K. Performance of the HIV Blot 2.2, INNO-LIA HIV I/II Score, and Geenius HIV 1/2 Confirmatory Assay for use in HIV confirmation. PLoS One. 2018;13:e0199502. https://doi.org/10.1371/ journal.pone.0199502 PMID:29928045.

13. Kondo M, Sudo K, Sano T, Kawahata T, Itoda I, Iwamuro S, et al. Comparative evaluation of the Geenius HIV 1/2 Confirmatory Assay and the HIV-1 and HIV-2 Western blots in the Japanese population. PLoS One. 2018; 13:e0198924. https://doi.org/10.1371/journal.pone.0198924 PMID:30379808.

14. Herssens N, Beelaert G, Fransen K. Discriminatory capacity between HIV-1 and HIV-2 of the new rapid confirmation assay Geenius. J Virol Methods. 2014;208:11-5. https://doi.org/10.1016/j.jviromet.2014.07.025 PMID:25075934.

15. Malloch L, Kadivar K, Putz J, Levett PN, Tang J, Hatchette TF, et al. Comparative evaluation of the Bio-Rad Geenius HIV-1/2 Confirmatory Assay and the Bio-Rad Multispot HIV-1/2 Rapid Test as an alternative differentiation assay for CLSI M53 algorithm-I. J Clin Virol. 2013;585:e85-e91. https://doi.org/10.1016/j.jcv.2013.08.008 PMID:24342484.

16. de Mendoza C, Lozano A, Caballero E, Cabezas T, Ramos J, Soriano V. Antiretroviral Therapy for HIV-2 Infection in Non-Endemic Regions. AIDS Rev. 2020;22:44-56. https://doi.org/10.24875/AIDSRev.M20000029 PMID: 32167506 .

\section{Publisher's Note}

Springer Nature remains neutral with regard to jurisdictional claims in published maps and institutional affiliations.

Ready to submit your research? Choose BMC and benefit from:
- fast, convenient online submission
- thorough peer review by experienced researchers in your field
- rapid publication on acceptance
- support for research data, including large and complex data types
- gold Open Access which fosters wider collaboration and increased citations
- maximum visibility for your research: over 100M website views per year
At BMC, research is always in progress.
Learn more biomedcentral.com/submissions

